\title{
The unscented filter as an alternative to the EKF for nonlinear state estimation: a simulation case study
}

\author{
Andrei Romanenko*, José A.A.M. Castro \\ Department of Chemical Engineering, University of Coimbra, Pinhal de Marrocos, Pólo II, 3030-290 Coimbra, Portugal
}

Received 20 March 2002; received in revised form 23 July 2003; accepted 23 July 2003

\begin{abstract}
The Kalman filter has been widely used for estimation and tracking of linear systems since its formulation in 1960 due to its simplicity and robustness. In many chemical engineering applications the extended Kalman filter (EKF) is often used to deal with certain classes of nonlinear systems. In spite of that, designing an EKF for highly nonlinear processes is not a trivial task, particularly those involving highly exothermic reactions. In this work we compared the performance of a new technique, the unscented filter, with that of the extended Kalman filter. The unscented filter produced better results without performing potentially ill-conditioned numerical calculations and linearly approximating the evolution of the state vector covariance.
\end{abstract}

(c) 2003 Elsevier Ltd. All rights reserved.

Keywords: Nonlinear dynamics; Mathematical modeling; Simulation; Nonlinear state estimation; Extended Kalman filter; Unscented filter

\section{Introduction}

In many engineering areas the extended Kalman filter (EKF) is widely used for state estimation and tracking. Numerous successful applications of this tool have been reported in the literature. Nonetheless, when one applies an EKF to a complex system, a few implementation and numerical problems may arise. One of them is the computation of the state transition matrix which calls for calculation of the Jacobian matrix and its matrix exponential. Besides being a computationally expensive operation, there is no universal and robust numerical way to carry it out (Moler \& Van Loan, 1978).

Moreover, the linearization at each time step can introduce large errors and even cause divergence of the filter (Wan \& van der Merwe, 2000). These concerns are especially acute in complex industrial set-ups (Wilson, Agarwal, \& Rippin, 1998). Although higher order Kalman filters exist, they are more difficult to implement and prone to instability. Grewal and Andrews (1993) introduced measures to improve numerical stability of the EKF while Mostov (1996) proposed a way to stabilize higher order EKF. In some applications

\footnotetext{
* Corresponding author. Tel.: +35-123-979-8700; fax: +35-123-979-8703.

E-mail address: andrew@eq.uc.pt (A. Romanenko).
}

(Chang \& Hwang, 1998a,b), however, when suboptimal filtering is justified, the original EKF algorithm can be modified in order to make it more robust in a nonlinear dynamic environment.

In his work, Schei (1997) proposed an improvement for the EKF in which using a central difference method it is possible to perturb the system and determine covariances without explicit calculation of the Jacobian.

Julier, Uhlmann, and Durrant-Whyte (1995) argued that with a fixed number of parameters it should be easier to approximate a Gaussian distribution than to approximate an arbitrary nonlinear function and introduced a new generalization of the Kalman filter, the unscented filter (UF), which permits the direct propagation of means and covariances through the nonlinear system equations. The main difference between the UF and the method developed by Schei (1997) is that the unscented filter approximates the underlying distribution.

In a striking review of new contributions in the field of state estimation, Nørgaard, Poulsen, and Ravn (2000) compared the two above mentioned filters and proposed a third formulation whose performance is argued to be superior to the filter described in (Schei, 1997) and similar to the one of the UF.

Despite the fact that these techniques apparently surpass the EKF, there have been no references in the literature to 
their application in the field of chemical engineering. In this work we apply the extended Kalman filter and the unscented filter to a highly nonlinear simulated CSTR in a system state estimation framework and compare their performance.

\section{The EKF and UF algorithms}

The linear filtering problem is concerned with the estimation of the state vector of a dynamic system, approximated in discrete time as

$\boldsymbol{x}_{k}=f\left(\boldsymbol{x}_{k-1}, \boldsymbol{u}_{k-1}, \boldsymbol{v}_{k-1}, k-1\right)$,

provided discrete observations, according to the measurement model

$\boldsymbol{y}_{k}=h\left(\boldsymbol{x}_{k}, \boldsymbol{u}_{k}, \boldsymbol{\omega}_{k}, k\right)$

where $\boldsymbol{x}$ is the state vector, $\boldsymbol{u}$ the control input vector, $\boldsymbol{v}$ the system noise vector, $\boldsymbol{\omega}$ the measurement noise vector, $f()$ the process model, and $h()$ is the measurement model.

The extended Kalman filter algorithm can be summarized as a series of the following steps:

$\hat{\boldsymbol{x}}_{k \mid k-1}=f\left(\hat{\boldsymbol{x}}_{k-1 \mid k-1}, \boldsymbol{u}_{k-1}, k-1\right)$,

$\boldsymbol{P}_{k \mid k-1}=\boldsymbol{\Phi}_{k-1} \boldsymbol{P}_{k-1 \mid k-1} \boldsymbol{\Phi}_{k-1}^{\mathrm{T}}+\boldsymbol{B}_{k-1} \boldsymbol{Q}_{k-1} \boldsymbol{B}_{k-1}^{\mathrm{T}}$,

$\hat{\boldsymbol{y}}_{k}=h\left(\hat{\boldsymbol{x}}_{k \mid k-1}, \boldsymbol{u}_{k-1}, k\right)$,

$\boldsymbol{K}_{k}=\boldsymbol{P}_{k \mid k-1} \boldsymbol{H}_{k}^{\mathrm{T}}\left[\boldsymbol{H}_{k} \boldsymbol{P}_{k \mid k-1} \boldsymbol{H}_{k}^{\mathrm{T}}+\boldsymbol{D}_{k} \boldsymbol{R}_{k} \boldsymbol{D}_{k}^{\mathrm{T}}\right]^{-1}$,

$\hat{\boldsymbol{x}}_{k \mid k}=\hat{\boldsymbol{x}}_{k \mid k-1}+\boldsymbol{K}_{k}\left(\boldsymbol{y}_{k}-\hat{\boldsymbol{y}}_{k}\right)$,

$\boldsymbol{P}_{k \mid k}=\left[I-\boldsymbol{K}_{k} \boldsymbol{H}_{k}\right] \boldsymbol{P}_{k \mid k-1}$,

provided

$\boldsymbol{\Phi}_{k-1}=\frac{\partial f}{\partial \boldsymbol{x}}, \quad \boldsymbol{B}_{k-1}=\frac{\partial f}{\partial \boldsymbol{v}}, \quad \boldsymbol{H}_{k}=\frac{\partial h}{\partial \boldsymbol{x}}, \quad \boldsymbol{D}_{k}=\frac{\partial h}{\partial \boldsymbol{\omega}}$.

In the equations above, $\boldsymbol{Q}$ and $\boldsymbol{R}$ are the covariance matrices of the process and measurement noises, respectively. More detailed information about the EKF algorithm, including different implementation equations and improvements, can be found elsewhere (Jazwinski, 1970; Grewal \& Andrews, 1993).

One of the main flaws of the EKF is the assumption that the errors in the state estimates are small and that the predicted mean of the state vector is equal to the prior mean projected through $f()$. It is also assumed that the state errors propagate through a separate linearized system (4). In a nonlinear environment these assumptions may undermine the filter performance.

In the unscented transformation (Julier et al., 1995; Julier \& Uhlmann, 1997), on which the UF is based, a set of weighed sigma points are deterministically chosen so that certain properties of these points match those of the prior distribution. Each point is then propagated through a nonlinear function and the properties of the transformed set are calculated. With this set of points, the unscented transform guarantees the same performance as the truncated second order Gaussian filter, with the same order of calculations as an extended Kalman filter but without the need to calculate Jacobians or Hessians (Julier, Uhlmann, \& Durrant-Whyte, 2000).

At first, the system state vector is augmented as

$\boldsymbol{x}_{k-1 \mid k-1}^{\mathrm{a}} \triangleq\left(\begin{array}{c}\boldsymbol{x}_{k-1 \mid k-1} \\ \boldsymbol{v}_{k-1} \\ \boldsymbol{\omega}_{k-1}\end{array}\right)$.

Its mean is

$E\left[\boldsymbol{x}_{k-1 \mid k-1}^{\mathrm{a}}\right]=\left(\begin{array}{c}\overline{\boldsymbol{x}}_{k-1 \mid k-1} \\ \mathbf{0}^{q \times 1} \\ \mathbf{0}^{r \times 1}\end{array}\right)$,

where $\mathbf{0}^{q \times 1}$ and $\mathbf{0}^{r \times 1}$ are vectors of zeroes and $q$ and $r$ are the sizes of the covariance matrices of the system and measurement noise, respectively.

The covariance of the augmented state vector is, therefore

$\boldsymbol{P}_{k-1 \mid k-1}^{a}=\left[\begin{array}{ccc}\boldsymbol{P}_{k-1 \mid k-1} & \boldsymbol{0}^{n \times q} & \boldsymbol{0}^{n \times r} \\ \mathbf{0}^{q \times n} & \boldsymbol{Q}_{k-1} & \boldsymbol{P}_{k-1}^{v \omega} \\ \boldsymbol{0}^{r \times n} & \boldsymbol{P}_{k-1}^{\omega \nu} & \boldsymbol{R}_{k-1}\end{array}\right]$,

where $n$ is the dimension of the original state vector, and $\boldsymbol{P}_{k-1}^{v \omega}$ and $\boldsymbol{P}_{k-1}^{\omega \nu}$ are the correlations between the system and measurement noises.

At the next step, a set of $2(n+q+r)+1$ symmetric sigma points is computed

$$
\begin{array}{r}
\mathcal{X}_{k-1 \mid k-1}^{*}=\left[\begin{array}{ll}
\mathbf{0}, & \sqrt{(n+q+r+\kappa) \boldsymbol{P}_{k-1 \mid k-1}^{\mathrm{a}}}, \\
-\sqrt{(n+q+r+\kappa) \boldsymbol{P}_{k-1 \mid k-1}^{\mathrm{a}}}
\end{array}\right],
\end{array}
$$

where $\kappa$ is a parameter for "fine tuning" the higher order moments of the approximation (Julier \& Uhlmann, 1997). For any symmetric prior distribution with kurtosis $k$, if $\kappa$ is chosen such that $0<n+q+r+\kappa \leq k$, then the predictions of means and covariances are more accurate than those of the EKF (Julier et al., 1995). For a Gaussian distribution, $n+q+r+\kappa=3$ should be used.

It is known that the matrix square root has no unique solution. Despite this fact, the algorithm properties presented above hold for any choice of its form. Julier et al. (1995) recommends the Cholesky decomposition as a robust and efficient method, which is of special importance in real-time applications.

The sigma point set $\mathcal{X}^{*}$ is zero mean and has the same variance as the augmented state vector $\boldsymbol{x}^{a}$. In order to correct the mean, the estimate $\hat{\boldsymbol{x}}_{k-1 \mid k-1}^{a}$ is added to each sigma point in vector $\mathcal{X}_{k-1 \mid k-1}^{*}$ and the resulting vector of sigma points is

$$
\begin{aligned}
& \mathcal{X}_{k-1 \mid k-1}=\left[\mathcal{X}_{k-1 \mid k-1,1}^{*}+\hat{\boldsymbol{x}}_{k-1 \mid k-1}^{a}, \ldots,\right. \\
& \left.\mathcal{X}_{k-1 \mid k-1,2 n^{\mathrm{a}}+1}^{*}+\hat{\boldsymbol{x}}_{k-1 \mid k-1}^{a}\right] \text {, }
\end{aligned}
$$


where $n^{a}$ is the dimension of the augmented state vector. In (13), the subscript $j$ of $\mathcal{X}_{k-1 \mid k-1, j}^{*}$ is the sigma point index. The state temporal update consists of two steps in which the vector $\mathcal{X}$ is mapped through the nonlinear system

$\mathcal{X}_{k \mid k-1}=f\left(\mathcal{X}_{k-1 \mid k-1}, u_{k-1}, k-1\right)$,

and the predicted mean is computed as

$\hat{\boldsymbol{x}}_{k \mid k-1}=\sum_{i=1}^{2 n^{a}+1} W_{i} \mathcal{X}_{i, k \mid k-1}^{a}$,

where $\boldsymbol{W}$ is a weight vector of size $2 n^{a}+1$ with elements

$W_{i}= \begin{cases}\frac{\kappa}{n^{\mathrm{a}}+\kappa}, & \text { if } i=1, \\ \frac{1}{2\left(n^{a}+\kappa\right)}, & \text { otherwise. }\end{cases}$

The predicted covariance is computed as

$$
\begin{aligned}
\boldsymbol{P}_{k \mid k-1}= & \sum_{i=1}^{2 n^{a}+1} W_{i}\left[\mathcal{X}_{i, k \mid k-1}-\hat{\boldsymbol{x}}_{k \mid k-1}\right] \\
& \times\left[\mathcal{X}_{i, k \mid k-1}-\hat{\boldsymbol{x}}_{k \mid k-1}\right]^{\mathrm{T}} .
\end{aligned}
$$

In a similar way the statistics for the innovation sequence must be determined. The mean observation is found from

$\mathcal{Y}_{k}=h\left(\mathcal{X}_{k \mid k-1}, u_{k}, k\right)$,

$\hat{\boldsymbol{y}}_{k}=\sum_{i=1}^{2 n^{a}+1} W_{i} \mathcal{Y}_{i, k}$,

and the covariance is determined as

$\boldsymbol{P}_{y}=\sum_{i=1}^{2 n^{a}+1} W_{i}\left[\mathcal{Y}_{i, k}-\hat{\boldsymbol{y}}_{k}\right]\left[\mathcal{Y}_{i, k}-\hat{\boldsymbol{y}}_{k}\right]^{\mathrm{T}}$.

The cross-correlation between the state estimate and the measurement sequence is, therefore,

$\boldsymbol{P}_{x y}=\sum_{i=1}^{2 n^{a}+1} W_{i}\left[\mathcal{X}_{i, k \mid k-1}-\hat{\boldsymbol{x}}_{k \mid k-1}\right]\left[\mathcal{Y}_{i, k}-\hat{\boldsymbol{y}}_{k}\right]^{\mathrm{T}}$.

At this point, the Kalman gain can be determined as

$\boldsymbol{K}_{k}=\boldsymbol{P}_{x y} \boldsymbol{P}_{y}^{-1}$,

the measurement update can be performed from

$\hat{\boldsymbol{x}}_{k \mid k}=\hat{\boldsymbol{x}}_{k \mid k-1}+\boldsymbol{K}_{k}\left(\boldsymbol{y}_{k}-\hat{\boldsymbol{y}}_{k}\right)$,

and the corresponding covariance matrix

$\boldsymbol{P}_{k \mid k}=\boldsymbol{P}_{k \mid k-1}-\boldsymbol{K}_{k} \boldsymbol{P}_{y} \boldsymbol{K}_{k}^{T}$.

\section{Application and results}

In order to evaluate the relative performance of the unscented filter in comparison to that of the EKF, they were
Table 1

Model parameters

\begin{tabular}{llll}
\hline Parameter & Expression & Value & Unit \\
\hline$p_{1}$ & $\frac{F_{\mathrm{R}, \mathrm{ref}}}{V_{\mathrm{R}}}$ & $3.333 \times 10^{-2}$ & $\mathrm{~s}^{-1}$ \\
$p_{2}$ & $k_{0} E_{\mathrm{a}}$ & $4.08 \times 10^{7}$ & $\mathrm{~s}^{-1}$ \\
$p_{3}$ & $\frac{E_{\mathrm{a}}}{R T_{\mathrm{R}, \mathrm{ref}}}$ & 25.347 & - \\
$p_{4}$ & $\frac{\left(h A_{T}\right)_{i}}{V_{\mathrm{R}}\left(\rho C_{p}\right)_{f}}$ & $6.63 \times 10^{-1}$ & $\mathrm{~s}^{-1}$ \\
$p_{5}$ & $\frac{-\left(\Delta H_{\mathrm{R}}\right)_{\mathrm{A}, \mathrm{ref}}}{\left(\rho C_{p}\right)_{f} T_{\mathrm{R}, \mathrm{ref}}}$ & 1.45 & - \\
$p_{6}$ & $\frac{\left(h A_{T}\right)_{i}}{V_{\mathrm{W}}\left(\rho C_{p}\right)_{\mathrm{W}}}$ & 5.97 & \\
$p_{7}$ & $\frac{\left(h A_{T}\right)_{e}}{V_{\mathrm{W}}\left(\rho C_{p}\right)_{\mathrm{W}}}$ & 5.97 & $\mathrm{~s}^{-1}$ \\
$p_{8}$ & $\frac{F_{\mathrm{J}, r e f}}{V_{\mathrm{J}}}$ & $1.67 \times 10^{-1}$ & $\mathrm{~s}^{-1}$ \\
$p_{9}$ & $\frac{\left(h A_{T}\right)_{e}}{V_{\mathrm{J}}\left(\rho C_{p}\right)_{\mathrm{J}}}$ & 1.33 & $\mathrm{~s}^{-1}$ \\
\hline
\end{tabular}

applied to a highly nonlinear dynamic system describing the behavior of a non-adiabatic CSTR in which an irreversible highly exothermic chemical reaction $(\mathrm{A} \rightarrow \mathrm{B})$ takes place. The reactor's wall significantly affects the system dynamics and therefore has also been taken into account. The corresponding model leads to the following set of ODEs in a normalized and dimensionless form:

$$
\left\{\begin{aligned}
\frac{\mathrm{d} x_{1}}{\mathrm{~d} t}= & p_{1} u_{1}+p_{1} u_{1} u_{2}-p_{1} u_{2} x_{1} \\
& -p_{2} \mathrm{e}^{-p_{3} /\left(1+x_{2}\right)}\left(1+x_{1}\right)-p_{1} x_{1}, \\
\frac{\mathrm{d} x_{2}}{\mathrm{~d} t}= & p_{1} u_{3}+p_{1} u_{2} u_{3}-p_{1} x_{2}-p_{1} u_{2} x_{2}-p_{4} x_{2} \\
& +p_{4} x_{3}+p_{5} p_{2} \mathrm{e}^{-p_{3} /\left(1+x_{2}\right)}\left(1+x_{1}\right), \\
\frac{\mathrm{d} x_{3}}{\mathrm{~d} t}= & p_{6} x_{2}-p_{6} x_{3}-p_{7} x_{3}+p_{7} x_{4}, \\
\frac{\mathrm{d} x_{4}}{\mathrm{~d} t}= & p_{8} u_{4}+p_{8} u_{4} u_{5}-p_{8} x_{4}-p_{8} u_{5} x_{4} \\
& +p_{9} x_{3}-p_{9} x_{4},
\end{aligned}\right.
$$

with the system state vector defined as

$$
\begin{aligned}
& \boldsymbol{x}^{\mathrm{T}}=\left[\begin{array}{ll}
\frac{C_{\mathrm{A}}-C_{\mathrm{A}, \mathrm{ref}}}{C_{\mathrm{A}, \text { ref }}} & \frac{T_{\mathrm{R}}-T_{\mathrm{R}, \mathrm{ref}}}{T_{\mathrm{R}, \mathrm{ref}}}
\end{array}\right. \\
& \left.\frac{T_{\mathrm{W}}-T_{\mathrm{W}, \text { ref }}}{T_{\mathrm{W}, \text { ref }}} \quad \frac{T_{\mathrm{J}}-T_{\mathrm{J}, \text { ref }}}{T_{\mathrm{J}, \text { ref }}}\right],
\end{aligned}
$$

where $C_{\mathrm{A}}$ is the concentration of reactant $\mathrm{A}, T_{\mathrm{R}}$ the reactor temperature, $T_{\mathrm{W}}$ the wall temperature, and $T_{\mathrm{J}}$ is the jacket

Table 2

Steady states

\begin{tabular}{lcrc}
\hline & Low-temperature stable & \multicolumn{1}{c}{ Unstable } & High-temperature stable \\
\hline$x_{1}$ & -0.0140582 & -0.37748 & -0.97640 \\
$x_{2}$ & 0.0068168 & 0.18304 & 0.47345 \\
$x_{3}$ & 0.0061321 & 0.16465 & 0.42590 \\
$x_{4}$ & 0.0054473 & 0.14627 & 0.37834 \\
\hline
\end{tabular}


temperature. The unit of $C_{\mathrm{A}}$ is $\mathrm{mol} / \mathrm{m}^{3}$ and the unit of $T_{\mathrm{R}}$, $T_{\mathrm{W}}$, and $T_{\mathrm{J}}$ is $\mathrm{K}$.

The input vector is

$$
\begin{aligned}
& \boldsymbol{u}^{\mathrm{T}}=\left[\frac{C_{\mathrm{A} 0}-C_{\mathrm{A}, \text { ref }}}{C_{\mathrm{A}, \text { ref }}} \frac{F_{\mathrm{R}}-F_{\mathrm{R}, \text { ref }}}{F_{\mathrm{R}, \text { ref }}}\right. \\
& \left.\frac{T_{0}-T_{0, \text { ref }}}{T_{0, \text { ref }}} \frac{T_{\mathrm{J} 0}-T_{\mathrm{J} 0 \text {,ref }}}{T_{\mathrm{J} 0 \text {,ref }}} \frac{F_{\mathrm{J}}-F_{\mathrm{J}, \text { ref }}}{F_{\mathrm{J} \text {,ref }}}\right] \text {, }
\end{aligned}
$$

where $C_{\mathrm{A} 0}$ is the feed concentration of reactant $\mathrm{A}, F_{\mathrm{R}}$ the flowrate, $T_{0}$ the feed temperature, $T_{\mathrm{J} 0}$ the inlet jacket tem- perature, and $F_{\mathrm{J}}$ is the coolant flowrate. The unit of $C_{\mathrm{A} 0}$ is $\mathrm{mol} / \mathrm{m}^{3}$ and the unit of $T_{0}$ and $T_{\mathrm{J} 0}$ is $\mathrm{K}$, and the unit of $F_{\mathrm{R}}$ and $F_{\mathrm{J}}$ is $\mathrm{m}^{3} / \mathrm{s}$. The corresponding reference values are: $C_{\mathrm{A} 0 \text {,ref }}=C_{\mathrm{A}, \text { ref }}=3 \mathrm{~mol} / \mathrm{m}^{3}, F_{\mathrm{R}, \text { ref }}=60 \times 10^{-5} \mathrm{~m}^{3} / \mathrm{s}$, $F_{\mathrm{J}, \text { ref }}=15 \times 10^{-4} \mathrm{~m}^{3} / \mathrm{s}, T_{\mathrm{R}, \text { ref }}=T_{\mathrm{W}, \text { ref }}=T_{\mathrm{J}, \text { ref }}=T_{0, \text { ref }}=$ $T_{\mathrm{J} 0 \text {, ref }}=298 \mathrm{~K}$.

The measurement model is assumed to be

$$
\boldsymbol{y}=\boldsymbol{H} \boldsymbol{x} \quad \text { with } \quad \boldsymbol{H}=\left[\begin{array}{llll}
1 & 0 & 0 & 0 \\
0 & 1 & 0 & 0 \\
0 & 0 & 0 & 1
\end{array}\right] \text {, }
$$


Fig. 1. Results of Test I (state $(x)$, output $(y)$, and the output setpoint $\left(y^{\mathrm{sp}}\right)$ are represented with solid, dashed, and dotted lines, respectively). 
that is, measurements of the wall temperature $x_{3}$ are not available.

Table 1 summarizes the model parameters used in the present work. The model has three steady states which are represented in Table 2 .

The objective of the present simulation is to compare the $\mathrm{UF}$ and the EKF in a nonlinear environment where the exact system and measurement models are available to the filters, as is the statistical information about the stochastic disturbances. Although such situation is oftentimes unrealistic in industrial practice, yet here it enables a fair comparison of such "ideally" tuned filters with no model mismatch affecting the results. For the purpose of evaluation the filters' behavior in a wide range of operating conditions, the simulation is performed in closed loop with clear intervals of regulatory and servo control and with the system state vector driven to each of the three steady states.

Three simulation tests are carried out with different measurement noise parameters. Each of the tests starts out with the state vector initialized at the high-temperature stable steady state. The reactor temperature $\left(y_{2}\right)$ is under control of two PI controllers working in a cascade: the first controls
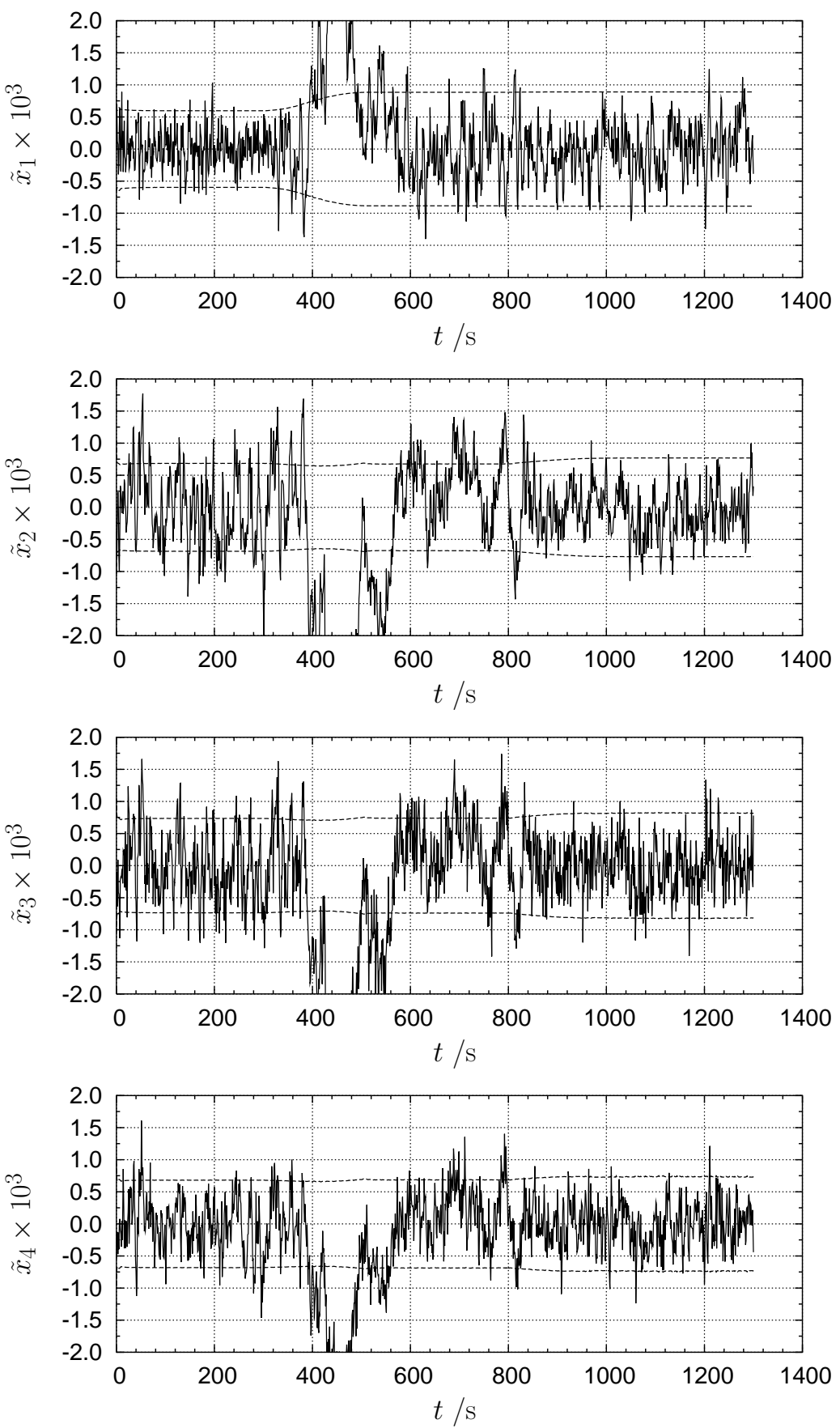

Fig. 2. Test II: EKF estimation errors and $2 \sigma$ bounds. 
the reactor temperature by manipulating the jacket temperature $\left(y_{3}\right)$ setpoint of the second PI controller, the latter manipulating the jacket flowrate $\left(u_{5}\right)$. In each experiment the reactor temperature setpoint is
The state vector $\boldsymbol{x}$ is subject to zero mean additive white noise with covariance

$\tilde{\boldsymbol{Q}}=\operatorname{diag}\left\{10^{-6}, 10^{-6}, 10^{-6}, 10^{-6}\right\}$,

$y_{2}^{\mathrm{sp}}= \begin{cases}0.47345, & \text { if } t \leq 300 \mathrm{~s}, \\ 0.47345-0.29041(t-300) / 200, & \text { if } 300 \mathrm{~s}<t \leq 500 \mathrm{~s}, \\ 0.18304, & \text { if } 500 \mathrm{~s}<t \leq 800 \mathrm{~s}, \\ 0.18304-0.1762232(t-800) / 200, & \text { if } 800 \mathrm{~s}<t \leq 1000 \mathrm{~s}, \\ 0.0068168, & \text { if } 1000 \mathrm{~s}<t \leq 1300 \mathrm{~s} .\end{cases}$

The measurement and control interval is $1 \mathrm{~s}$.


Fig. 3. Test II: UF estimation errors and $2 \sigma$ bounds. 
while the measurements are corrupted by zero mean additive white noise with covariance

$$
\tilde{\boldsymbol{R}}= \begin{cases}\operatorname{diag}\left\{10^{-8}, 10^{-8}, 10^{-8}\right\} & \text { in Test I, } \\ \operatorname{diag}\left\{6.5 \times 10^{-7}, 6.5 \times 10^{-7}, 6.5\right. & \\ \left.\times 10^{-7}\right\} & \text { in Test II, } \\ \operatorname{diag}\left\{10^{-5}, 10^{-5}, 10^{-5}, 10^{-5}\right\} & \text { in Test III. }\end{cases}
$$

The simulation results of Test I are illustrated in Fig. 1. For the sake of space, we omit the respective graphics of Tests II and III as the state vector and outputs behave similarly.
The EKF and UF filter parameters are

$$
\begin{aligned}
\hat{\boldsymbol{x}}_{0} & =\boldsymbol{x}_{0}+\left[10^{-3},-10^{-3}, 10^{-3}, 10^{-3}\right]^{\mathrm{T}} \\
& =[-0.97540,0.47245,0.42690,0.37934]^{\mathrm{T}}, \\
\hat{\boldsymbol{P}}_{0} & =\operatorname{diag}\left\{10^{-6}, 10^{-6}, 10^{-6}, 10^{-6}\right\}, \\
\boldsymbol{Q} & =\tilde{\boldsymbol{Q}} \quad \text { and } \quad \boldsymbol{R}=\tilde{\boldsymbol{R}} .
\end{aligned}
$$

Additionally, parameter $\kappa$ of the UF is set to -5 based on the dimension of the state vector and the characteristics of
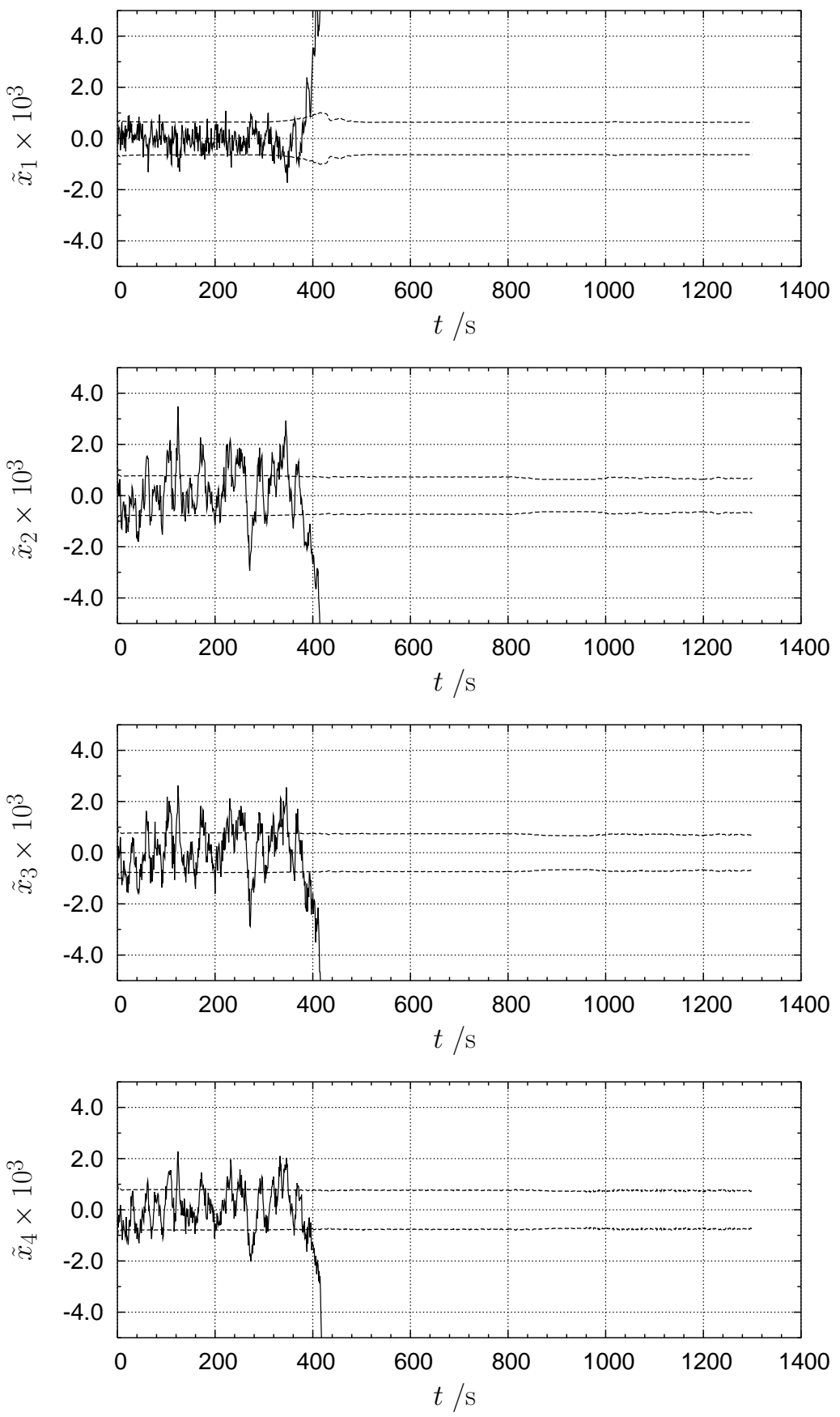

Fig. 4. Test III: EKF estimation errors and $2 \sigma$ bounds. 
the noises. We emphasize that no further adjustment of $\kappa$ with the purpose of improving UF performance took place. Finally, it is worth mentioning that the filter parameters are consistent and reflect the parameters of the actual noise sources acting on the system.

In order to decrease estimation errors of the EKF algorithm, the predicted state estimate (3) was computed using direct numerical integration of the state equations.

The profiles of the estimation errors (residuals) of both filters and the respective $2 \sigma$ bounds in single runs (calcu- lated as twice the square root of the trace of the state covariance matrix) are illustrated in Figs. 2-5. For the purpose of ensuring a fair comparison of the filtering techniques under consideration, a Monte-Carlo simulation of 50 runs was carried out for each set of noise parameters with a consequent step of averaging the results. The overall estimation errors are summarized in Table 3. A description of the filtering results obtained in each test follows.

Test I: Since the measurement noise covariance is small as compared to the dynamics of the system, the estimation
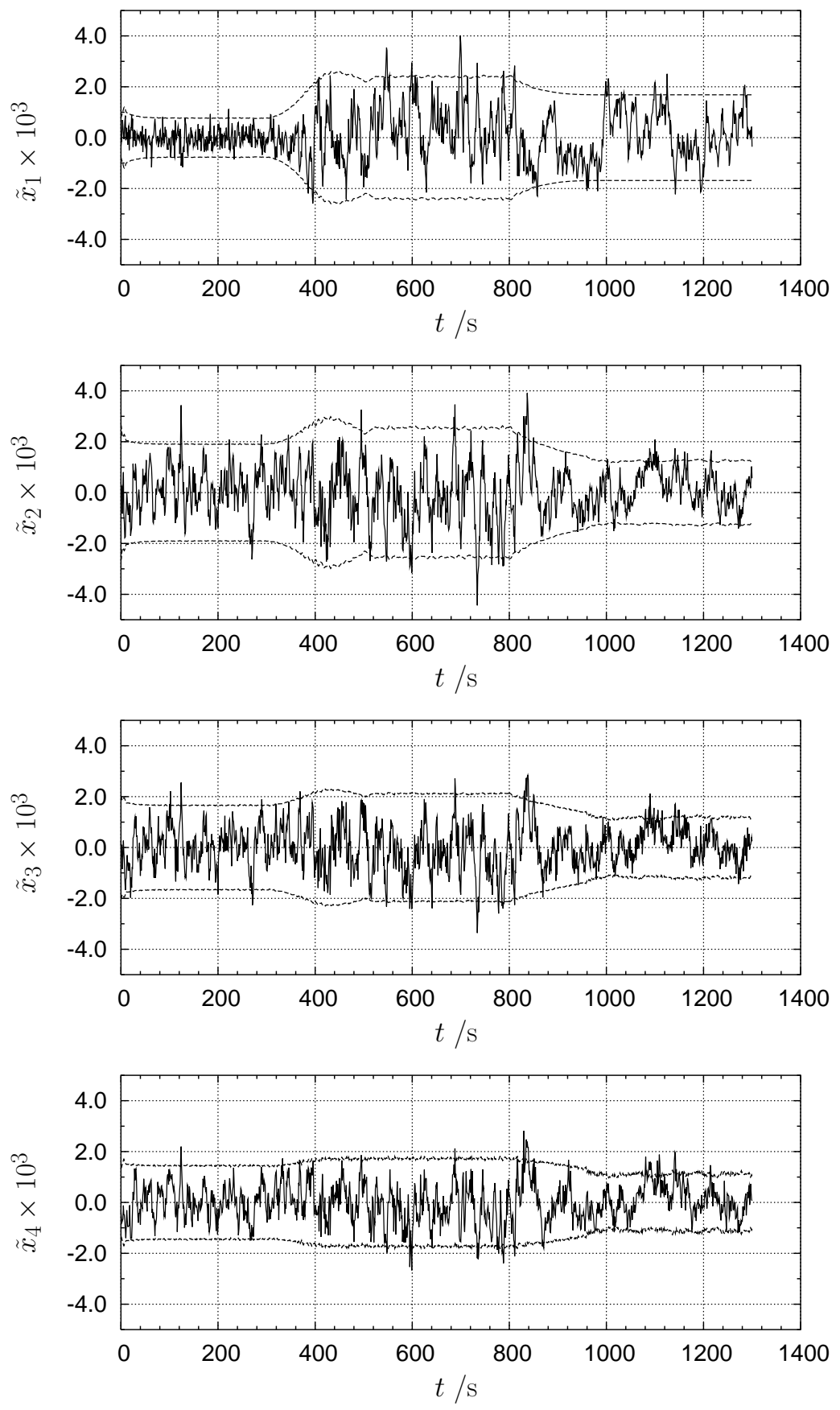

Fig. 5. Test III: UF estimation errors and $2 \sigma$ bounds. 
Table 3

Mean square estimation errors over 50 runs

\begin{tabular}{|c|c|c|c|c|}
\hline Method & $x_{1}$ & $x_{2}$ & $x_{3}$ & $x_{4}$ \\
\hline \multicolumn{5}{|c|}{ Test I: $\boldsymbol{R}=\operatorname{diag}\left\{10^{-8}, 10^{-8}, 10^{-8}\right\}$} \\
\hline $\mathrm{EKF}$ & $1.1918 \times 10^{-5}$ & $1.1993 \times 10^{-5}$ & $1.3878 \times 10^{-4}$ & $1.1808 \times 10^{-5}$ \\
\hline UF & $1.1918 \times 10^{-5}$ & $1.1986 \times 10^{-5}$ & $1.3830 \times 10^{-4}$ & $1.1806 \times 10^{-5}$ \\
\hline \multicolumn{5}{|c|}{ Test II: $\boldsymbol{R}=\operatorname{diag}\left\{6.5 \times 10^{-7}, 6.5 \times 10^{-7}, 6.5 \times 10^{-7}\right\}$} \\
\hline EKF & $5.3636 \times 10^{-4}$ & $1.0863 \times 10^{-3}$ & $8.9844 \times 10^{-4}$ & $4.3803 \times 10^{-4}$ \\
\hline UF & $2.2175 \times 10^{-4}$ & $2.7366 \times 10^{-4}$ & $2.6355 \times 10^{-4}$ & $1.8314 \times 10^{-4}$ \\
\hline \multicolumn{5}{|c|}{ Test III: $\boldsymbol{R}=\operatorname{diag}\left\{10^{-5}, 10^{-5}, 10^{-5}\right\}$} \\
\hline EKF & 200.310 & 71.636 & 60.324 & 48.166 \\
\hline UF & $1.0423 \times 10^{-3}$ & $1.3258 \times 10^{-3}$ & $9.7272 \times 10^{-4}$ & $7.0139 \times 10^{-4}$ \\
\hline
\end{tabular}

errors of the EKF and the UF are essentially the same (Table 3), that is, although there may exist linearization errors in the calculation of the state transition matrix in the EKF algorithm, they do not have significant impact on the filter performance as the estimates are mostly based on measurements. However, even in this case the UF may be preferred to the EKF because it needs the system and measurement models only and does not require derivative information. Because of the similar performance of EKF and UF in this test, the corresponding graphic of filtering residuals is omitted.

Test II: As the measurement noise increases, the filters tend to weigh more the a priori estimate obtained using the model. In this case, the linearization errors of the EKF begin to play a significant role and the estimation residuals of the EKF are from two to five times greater than the ones of the UF (Table 3). Analyzing the $2 \sigma$ bounds in Fig. 2 one can observe that the EKF overestimates the state covariance and this leads to inconsistent results. On the contrary, the residuals produced by the UF lie within the $2 \sigma$ interval most of the time (Fig. 3).

Test III: In this extreme case the measurement noise is very significant and the filter estimates are based mostly on the a priori estimate. The linearization errors of the EKF impact severely its state estimates and ultimately lead to its divergence (Fig. 4). However, the UF continues operating satisfactorily (Fig. 5).

\section{Conclusions}

In this paper, we have assessed the use of the unscented filter in a typical chemical engineering application and found out that this estimation tool is able to provide superior accuracy in comparison to the ones produced by the EKF. The absence of model linearization step and, consequently, errors induced by it, allows the UF to be used in a wider range of operating conditions as well as in situations where the differentiability of the system model cannot be assured. Furthermore, the derivative free design of the UF makes its implementation significantly simpler than the one of the EKF.

\section{Acknowledgements}

This work had financial support from Program PRAXIS in the form of a scholarship for the first author (PRAXIS XXI/BD/19609/99). The authors are grateful to Simon Julier for his assistance in the theory of UF. The efforts of John W. Eaton and the other developers of Octave, a high-level computer language used in this work, are acknowledged.

\section{References}

Chang, C.-T., \& Hwang, J.-I. (1998a). Simplification techniques for EKF computations in fault diagnosis-suboptimal gains. Chemical Engineering Science, 53(22), 3853-3862.

Chang, C.-T., \& Hwang, J.-I. (1998b). Simplification techniques for EKF computations in fault diagnosis: model decomposition. AIChE Journal, 44(6), 1392-1403.

Grewal, M. S., \& Andrews, A. P. (1993). Kalman filtering: Theory and practice. Englewood Cliffs, NJ: Prentice-Hall.

Jazwinski, A. H. (1970). Stochastic processes and filtering theory. New York: Academic Press.

Julier, S. J., \& Uhlmann, J. K. (1997). A new extension of the Kalman filter to nonlinear systems. In Proceedings of AeroSense: The 11th International Symposium on Aerospace/Defense Sensing, Simulation and Controls (pp. 182-193). Orlando, FL, USA: SPIE.

Julier, S. J., Uhlmann, J. K., \& Durrant-Whyte, H. F. (1995). A new approach for filtering nonlinear systems. In Proceedings of the 1995 American Control Conference (pp. 1628-1632).

Julier, S. J., Uhlmann, J., \& Durrant-Whyte, H. F. (2000). A new method for the nonlinear transformation of means and covariances in filters and estimators. IEEE Transactions on Automatic Control, 45(3), 477-482.

Moler, C., \& Van Loan, C. (1978). Nineteen dubious ways to compute the exponential of a matrix. SIAM Review, 20, 801-836.

Mostov, K. (1996). Fuzzy adaptive stabilization of higher order Kalman filters. Master's thesis. Berkley: University of California.

Nørgaard, M., Poulsen, N. K., \& Ravn, O. (2000). Advances in derivative-free state estimation for nonlinear systems. Automatica, 36(11), 1627-1638.

Schei, T. S. (1997). A finite difference method for linearization in nonlinear estimation algorithms. Automatica, 33(11), 2051-2058.

Wan, E. A., \& van der Merwe, R. (2000). The unscented Kalman filter for nonlinear estimation. In Proceedings of Symposium 2000 on Adaptive Systems for Signal Processing, Communication, and Control (AS-SPCC) (pp. 153-158). Lake Louise, Alberta, Canada: IEEE.

Wilson, D. I., Agarwal, M., \& Rippin, D. W. T. (1998). Experiences implementing the extended Kalman filter on an industrial batch reactor. Computers Chemical Engineering, 22(11), 1653-1672. 